\title{
Invasiveness of Phragmites australis in communities dominated by native species after fire disturbance under controlled conditions
}

\author{
María Elena Páramo Pérez ${ }^{1}$, Roberto Lindig-Cisneros² y Patricia Moreno-Casasola ${ }^{3}$ \\ ${ }_{1}^{1}$ Posgrado en Ciencias Biológicas, Universidad Nacional Autónoma de México. Edificio D, primer piso, Circuito de Posgrados, Ciudad Universitaria, Coyoacán, Ciudad de \\ México, 04510. México \\ ${ }^{2}$ Laboratorio de Restauración Ambiental, Instituto de Investigaciones en Ecosistemas y Sustentabilidad, Universidad Nacional Autónoma de México, Campus Morelia. \\ Antigua carretera a Pátzcuaro núm. 8701, Morelia, Michoacán, 58190, México \\ ${ }^{3}$ Red de Ecología Funcional. Instituto de Ecología, A.C. Apdo. 63, Xalapa, Veracruz, 91000. México \\ e-mail: rlindig@iies.unam.mx
}

Recibido: 05 de diciembre de $2016 . \quad$ Aceptado: 11 de mayo de 2018.

Páramo Pérez M. E., R. Lindig-Cisneros y P. Moreno-Casasola. 2018. Potencial de invasión de Phragmites australis en comunidades dominadas por especies nativas ante disturbios por fuego en condiciones controladas. Hidrobiológica 28 (1): 201-207. DOI: 10.24275/uam/izt/dcbs/hidro/2018v28n2/Lindig

\section{RESUMEN}

Antecedentes. Los humedales son ecosistemas heterogéneos y dinámicos muy susceptibles a sufrir invasiones 0 extinciones locales por especies invasoras o sobredominantes. La resistencia a la invasión de los humedales ante factores de disturbio como el fuego ha sido poco estudiada. Cuando Phragmites australis (carrizo) se encuentra presente, su interacción con factores de disturbio ha llevado a la extinción local de muchas especies nativas. En un estudio anterior se determinó que la cosecha de esta especie es una estrategia efectiva de control. Sin embargo, la remoción de las partes aéreas genera múltiples fragmentos a partir de los cuales se podría propagar la especie. Objetivos. Evaluar, ante disturbios ocasionados por el fuego, en condiciones controladas, el potencial de invasión de fragmentos de Phragmites australis en comunidades dominadas por especies nativas. Métodos. El experimento se efectuó bajo dos quemas en 36 mesocosmos con doseles de Schoenoplectus americanus y $P$. australis. Resultados. Los resultados establecieron que las plantas de Phragmites australis no lograron desarrollarse después de las quemas del dosel de $S$. americanus, debido a la rápida recuperación del dosel de esta segunda especie. Se registró mayor supervivencia y crecimiento en los mesocosmos con bajo disturbio ( $27.7 \%$ y $55.9 \mathrm{~cm}$ en el primer ensayo; $9.4 \%$ y $60.6 \mathrm{~cm}$ en el segundo ensayo) en comparación con los controles $(8.5 \%, 35.3 \mathrm{~cm}$ y $7.4 \%$ y $86.7 \mathrm{~cm})$, ya que el dosel de $S$. americanus se mantuvo como una barrera al desarrollo de $P$. australis en los controles. Las diferencias en la altura fueron significativas después del primer evento de fuego entre los mesocosmos quemados y los de control $(p=0.002)$, pero después de dos años de quema no lo fueron ( $p$ $=0.085$ ), debido a que las pocas plantas que sobrevivieron en los mesocosmos control alcanzaron alturas considerables. Conclusiones. Los fragmentos de $P$. australis representan bajo riesgo para sitios con dosel de $S$. americanus después de un año de registrar disturbios como el fuego.

Palabras clave: plantas invasoras, dosel, Phragmites australis, regeneración, Schoenoplectus americanus

\begin{abstract}
Background. Wetlands are heterogeneous and dynamic ecosystems, very susceptible to invasions or local extinctions by the effects of invasive or over-dominant plant species. Resistance to invasion in wetlands that suffer disturbances such as fires has not been thoroughly studied. When Phragmites australis (common read) is present, its interaction with disturbance factors has led to local extinction of many native species. In a previous study, it was determined that harvesting is an effective control method for this species. But removal of aerial parts of this species generates many fragments that might propagate the species. Goals. Evaluate under controlled conditions the invasive potential of fragments of Phragmites australis in plant communities dominated by native plant species that are subjected to frequent human-made disturbances, such as fire, under controlled conditions. Methods. We carried out an experiment that consisted of two assays in 36 mesocosms with canopies of Schoenoplectus americanus. Results. Phragmites australis was practically not able to establish itself after the loss of the $S$. americanus canopy following fires, because the canopy of this last species
\end{abstract}


recovered rapidly. Survival and growth were slightly higher in mesocosms with low disturbance $(27.7 \%$ and $55.9 \mathrm{~cm}$ in the first assay, and $9.4 \%$, and $60.6 \mathrm{~cm}$ in the second assay), when compared with controls $(8.5 \% 35.3 \mathrm{~cm}$, and $7.4 \%$ and $86.7 \mathrm{~cm}$ ), because in control units the canopy of $S$. americanus was a permanent barrier to the development of $P$. australis. Height differences among burned and control mesocosms after the first fire event were significant $(p=0.002)$ but not after two consecutive years of burning $(p=0.085)$, because the few plants that survived in control units reached considerable height. Conclusions. The risk of establishment of the fragments of Phragmites australis that are generated during harvesting is low even after major disturbances of native vegetation cover.

Keywords: canopy, invasive control, Phragmites australis, regeneration, Schoenoplectus americanus

\section{INTRODUCCIÓN}

Los humedales son ecosistemas que abarcan diversos tipos de vegetación con una gran biodiversidad; sus características hidrobiológicas dan lugar a lagunas someras, humedales herbáceos de agua dulce, marismas, manglares, pantanos y selvas inundables, entre otros (Moreno-Casasola et al., 2010). Todos estos tipos de humedales han sido alterados drásticamente alrededor del mundo, lo cual ha causado pérdidas de biodiversidad y de servicios ecosistémicos (Constanza et al., 1997; MEA, 2005; Korsgaard \& Schou, 2010). Landgrave y MorenoCasasola (2012) reportan en México una pérdida o degradación de humedales del $62.1 \%$.

Las comunidades vegetales con una fisonomía particular que conforman estos ecosistemas, además de ser el componente biótico distintivo de los humedales, hacen posible el funcionamiento ecosistémico que los distingue de los ecosistemas terrestres (Mitsch \& Gosselink, 2000; Moreno-Casasola et al., 2010), ya que participan como base de la cadena alimentaria y proporcionan el hábitat para una variedad de organismos acuáticos, anfibios y terrestres, que incluye macroinvertebrados, bacterias epífitas, perifiton, aves, peces, reptiles, anfibios y aves (Zedler \& Kercher, 2005; Mitsch \& Gosselink, 2000). La vegetación influye en el régimen hidrológico, los procesos de sedimentación y la dinámica de los nutrientes (Zedler \& Kercher, 2005; Korsgaard \& Schou, 2010).

Entre los diferentes disturbios naturales que los impactan, el fuego se considera generalmente infrecuente, debido a los altos niveles de humedad y a las diferencias en los potenciales combustibles respecto a los ecosistemas terrestres (Miller et al., 1998; Bisson et al., 2003; Pettit \& Naiman, 2007). Sin embargo, los rayos (Bisson et al., 2003), la combustión espontánea (Miller et al., 1998; Bisson et al., 2003) y los incendios naturales en las partes altas de los ecosistemas pueden propagarse hacia los humedales y hacer posible la presencia del fuego en algunos de ellos, o bien aumentar su extensión e intensidad en aquéllos con un régimen natural de incendios (Pettit \& Naiman, 2007; Norton \& De Lange, 2002; Miller et al., 1998). Asimismo, diversos humedales son sometidos a regímenes de disturbio por fuego provocados por el ser humano (Bixby et al., 2015).

Un disturbio como el fuego modifica la composición y estructura de las comunidades vegetales a través de las respuestas y características funcionales de las especies presentes en el sitio. Además, el fuego interacciona con otros tipos de disturbio, como cambios en el hidroperiodo o la introducción de especies exóticas (Zedler \& Kercher, 2004; López Rosas \& Moreno-Casasola, 2012).
En las comunidades vegetales se ha documentado la modificación de las interrelaciones entre las especies como uno de los efectos del disturbio por fuego. Por ejemplo, en los Everglades, en los Estados Unidos, donde coinciden Typha domingensis Pers. y Phragmites australis (Cav.) Trin ex Stuedel, la primera puede llegar a desplazar a la segunda -al menos temporalmente- en zonas con un régimen de fuegos que presentan alteraciones en su hidrología (Newman et al., 1996; David, 1996; Miller et al., 1998; Rice et al., 2000; Pettit \& Naiman, 2007). En otros casos, la combinación de inundación y aumento en la salinidad tras un incendio puede disminuir significativamente la cobertura de $P$. australis (Rice et al., 2000; Bart \& Hartam, 2003). Diversos estudios en humedales riparios han demostrado que $P$. australis y Tamarix ramossisima Ledeb crean una sinergia con el fuego debido a que acumulan una gran cantidad de materia orgánica en pie que favorece incendios de alta intensidad con un mayor tiempo de residencia, lo cual deposita mayor cantidad de fósforo en el agua y, por lo tanto, elimina a otras especies vegetales más sensibles al fuego (Mitsch \& Gosselink, 2000; Zedler \& Kercher, 2005; Pettit \& Naiman, 2007). Lo anterior indica que la respuesta de la vegetación y de las especies de los humedales al fuego es idiosincrática, lo que obliga a estudiar la relación de este con cada tipo de humedal.

En un estudio anterior realizado por Escutia-Lara et al. (2012) fue posible determinar que la cosecha de Phragmites australis puede ser una medida efectiva de control, particularmente útil en sitios en donde el uso de herbicidas no es recomendable. Sin embargo, la cosecha produce múltiples fragmentos de longitudes pequeñas que no es posible retirar del sitio y que pueden dar origen a nuevos individuos y propagar la especie a zonas no invadidas dentro del humedal manejado. Debido a esto, se llevó a cabo un experimento en mesocosmos, con el objetivo de cuantificar el éxito en el establecimiento de fragmentos de $P$. australis bajo doseles de Schoenoplectus americanus (Pers.) Volkart ex Schinz \& R. Keller (una especie dominante de humedales del occidente de México) sometidos al efecto del fuego. En él se buscó comprobar si el fuego es un factor que favorece el establecimiento de $P$. australis, al reducir la competencia con Schoenoplectus americanus.

\section{MATERIALES Y MÉTODOS}

Descripción del sistema de referencia y las especies utilizadas. Los propágulos de Phragmites australis fueron colectados en el manantial de la Mintzita, sitio RAMSAR (101 $\left.17^{\prime} 47^{\prime \prime} 019^{\circ} 38^{\prime} 43^{\prime \prime} \mathrm{N}\right)$, ubicado al sur de la ciudad de Morelia, Michoacán, México, cuyo ecosistema está dominado por Typha domingensis y Schoenoplectus americanus (Hall et al., 2008; Escutia et al., 2009; del Val et al., 2012). Este humedal se ve afectado por fuertes presiones de actividad humana: provee alrede- 
dor del $35 \%$ de la totalidad de agua de la ciudad, presenta procesos de eutrofización, ramoneo de la vegetación por ganado y disturbio por fuegos que escapan de campos de cultivo aledaños (Escutia et al., 2009; Escutia et al., 2012). Asimismo, las especies nativas sufren la presión de plantas invasoras como Phragmites australis, pues éstas han empezado a formar manchones que dominan algunas partes del humedal.

Las especies dominantes en este sistema son comunes para la mayoría de los humedales templados de México. En las partes menos profundas se encuentra Schoenoplectus americanus (de la familia Cyperaceae), que es una planta perenne rizomatosa con un tallo erecto triangular que mide hasta $1.5 \mathrm{~m}$ de altura. Esta especie se distribuye en las regiones templadas desde Estados Unidos hasta Venezuela y Chile (Moreno-Casasola et al., 2010; Zedler \& Kercher, 2005). En el sitio de colecta de plántulas -que hemos estudiado por más de diez años- se encuentra regularmente más cerca a la orilla del humedal, en donde el agua se encuentra por debajo de la superficie del suelo (Escutia et al., 2009; Escutia et al., 2012).

Por otro lado, Phragmites australis es una planta de la familia Poaceae con una amplia distribución geográfica, desde zonas templadas a tropicales. Crece de dos a cuatro metros de altura y presenta hojas lisas y planas de 1 a $5 \mathrm{~cm}$ de ancho. Además, se propaga fácilmente por medio de estolones, rizomas y fragmentos de tallos (Escutia-Lara et al., 2012; Bart \& Hartman, 2003) y forma conjuntos densos que excluyen a otras especies vegetales (Swearinger \& Saltonstall, 2010)

Diseño experimental. Construcción de los mesocosmos. Se construyeron 36 mesocosmos con tinas de plástico de $210 \mathrm{~L}$ de capacidad $(100 \times 68 \times 40 \mathrm{~cm})$ con un área superficial de $0.58 \mathrm{~m}^{2}$. Cada mesocosmo fue llenado con una mezcla de turba comercial y suelo de la localidad (tipo arcilloso) con una proporción de 1:1 (0.1 m³ por material) (Fig. 1). El sistema de riego mantenía un nivel constante de agua, $5 \mathrm{~cm}$ por debajo de la superficie del sustrato. Los mesocosmos se encontraban al aire libre, sometidos a las variaciones naturales del clima

Fase 1. Plantado y desarrollo del dosel. Se efectuó el plantado de fragmentos de rizoma de Schoenoplectus americanus en los mesocosmos (16 por mesocosmo), y se dejó desarrollar un dosel de esta especie durante dos años, de 2011 a 2012 (Fig. 1). Este periodo de acondicionamiento permitió validar que no hubo diferencias entre los 36 mesocosmos en la densidad de tallos de Schoenoplectus americanus al final del periodo de acondicionamiento $(p>0.05)$.

Fase 2. Primera aplicación de fuego en el bioensayo. El primer tratamiento de quemado se aplicó durante la primera semana del mes de abril del 2013 a 18 unidades experimentales (la mitad) seleccionadas al azar. Cada mesocosmo fue asignado a un tratamiento por medio de una tabla de números aleatorios (Fig. 2). Una vez quemados los doseles de los mesocosmos asignados a este tratamiento, se trasplantaron dos segmentos vegetativos de Phragmites australis en cada uno de los 36 mesocosmos, con un total de 72 fragmentos. Los fragmentos, que eran de $30 \mathrm{~cm}$ de longitud y $8 \pm 2 \mathrm{~mm}$ de diámetro cada uno, fueron enraizados previamente en agua corriente en las instalaciones del Instituto de Investigaciones en Ecosistemas y Sustentabilidad durante 30 días (Fig. 1). Se plantaron en la parte central de cada mesocosmo a una distancia de $36 \mathrm{~cm}$ uno del otro por medio de una varilla de metal, a fin de causar el menor daño posible al sistema radicular de las plantas circunstantes

Fase 3. Segunda aplicación de fuego en el bioensayo. En el año 2014, durante la primera semana del mes de abril, se quemaron al azar 9 de los 18 mesocosmos que fueron quemados el año anterior (Figs. 1-2). Debido a que se pudo determinar que no había riesgo de competencia entre los segmentos de Phragmites durante una sola temporada de crecimiento, para esta aplicación se incluyó un mayor número de segmentos, ya que se pudieron plantar con menor distancia, de tal forma que se pudo incrementar el tamaño de muestra.

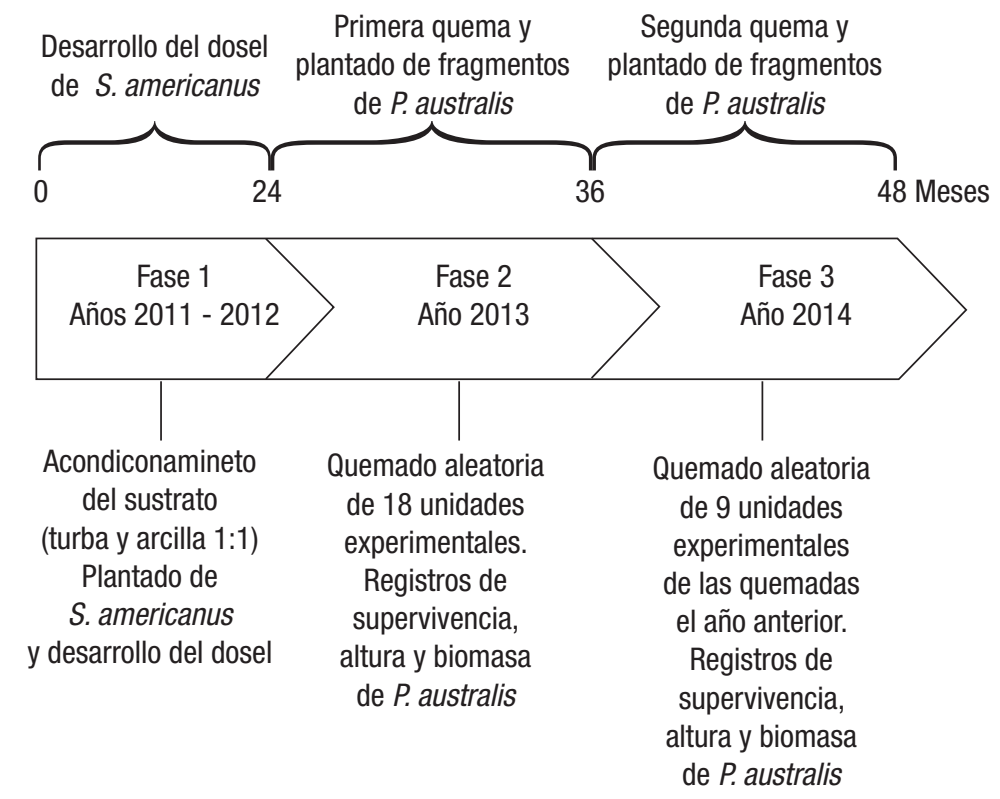

Figura 1. Línea del tiempo con descripción de las fases del experimento del establecimiento de fragmentos de Phragmites australis (Cav.) Trin ex Stuedel en un dosel de Schoenoplectus americanus (Pers.) Volkart ex Schinz \& R. Keller a lo largo de 4 años. 


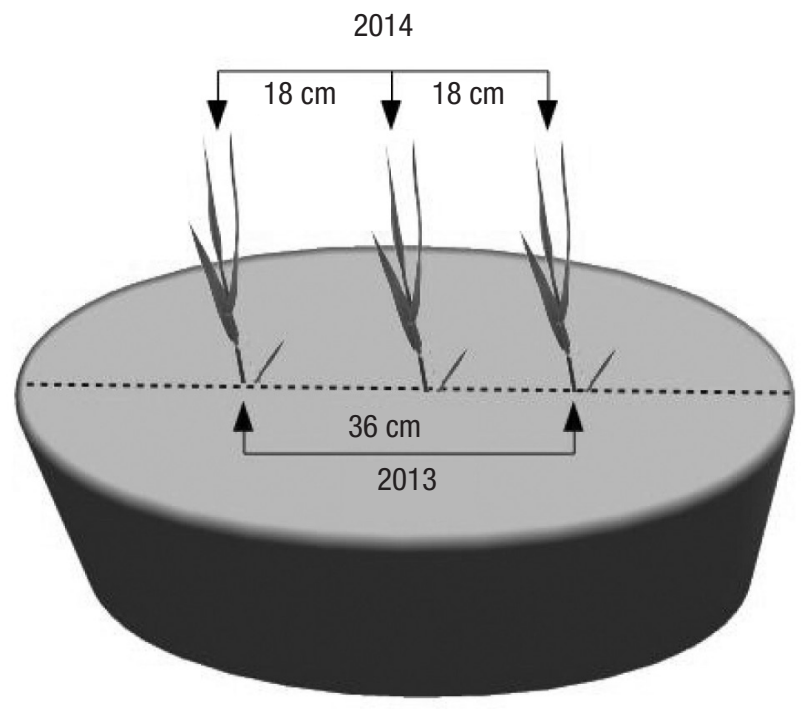

Figura 2. Esquema del plantado de los fragmentos de Phragmites australis (Cav.) Trin ex Stuedel los mesocosmos. Para el primer año se plantaron dos individuos por unidad y, al no haber evidencia de interferencia entre las plantas, para el segundo año se plantaron tres.

Respuesta de Phragmites australis: supervivencia, crecimiento y acumulación de biomasa. En ambas temporadas de crecimiento (de abril a octubre de 2013 y 2014) se registró la supervivencia y la altura máxima de los 36 mesocosmos (se utilizó una regla graduada cada 0.5 $\mathrm{cm})$. Para obtener los datos de biomasa aérea se colectaron los individuos de Phragmites australis en la última semana del mes de octubre; se colocaron en un horno de secado (Lumistel HEC-41) a $60^{\circ} \mathrm{C}$, hasta peso constante (lo que para la mayoría de las muestras ocurrió a las 48 horas), y se pesaron en una balanza semianalítica (Ohaus AV53). EI dosel de Schoenoplectus americanus no se evaluó a lo largo del experimento debido a su rápida recuperación.

Análisis estadístico. Los datos fueron analizados por medio de modelos lineales generalizados para supervivencia usando la distribución binomial. Para las variables continuas (altura y biomasa) se utilizó la distribución Poisson (Crawley, 2007), debido a que no cumplían con los supuestos asociados a la distribución normal. Todos los análisis estadísticos se llevaron a cabo con el paquete R (R Core Team, 2017).

\section{RESULTADOS}

Al inicio del experimento los mesocosmos mantuvieron una densidad de $11.0 \pm 0.6$ tallos $/ \mathrm{dm}^{2}$ de Schoenoplectus americanus, antes de efectuar la primera quema ( $p=0.55)$, lo que garantizó condiciones ambientales similares en los 36 mesocosmos. Cabe destacar que antes de las quema todos los mesocosmos presentaron rebrotes de Schoenoplectus americanus; el registro de su aparición inicio del mes de febrero de cada año y alcanzó la máxima cobertura del dosel en la segunda semana de agosto de 2013, aproximadamente cuatro meses después de aplicar el fuego durante la primera semana del mes de abril.
Posterior a la primera quema, en 2013, la supervivencia de las plantas de $P$. autralis fue de $27.8 \%$, mayor a lo detectado en los mesocosmos control, de 8.6\%. Después de la segunda quema, en 2014, en los mesocosmos quemados sólo en ese año la supervivencia fue de $7.4 \%$, de $9.4 \%$ para los mesocosmos quemados por dos años consecutivos y nula para los mesocosmos control.

En términos del incremento de altura de $P$. australis, al final de la temporada de crecimiento posterior a la primera quema, en 2013, el crecimiento en los mesocosmos sometidos al fuego fue de $55.9 \pm 36.7$ $\mathrm{cm}$, valor promedio mayor a lo detectado en los mesocosmos control, que fue de $35.3 \pm 6.2 \mathrm{~cm}$. Estas diferencias fueron validadas mediante un modelo lineal generalizado para los mesocosmos en donde había plantas vivas ( $Z=3.12 ; p=0.002 ; n=13)$. Para el segundo año de quemas, 2014, el crecimiento de los mesocosmos sometidos al fuego por un solo año dio como resultado alturas promedio de $86.76 \mathrm{~cm}$, valor promedio mayor al de los mesocosmos quemados en dos años consecutivos, que fue de $60.6 \pm 10.7 \mathrm{~cm}$ en promedio, y no hubo registros de altura para los mesocosmos control debido a que todos los individuos murieron. La diferencia en altura para las plantas de mesocosmos quemados un año y dos años no fue estadísticamente significativa $(Z=$ -1.726; $p=0.085 ; \mathrm{n}=9$ ) (Fig. 4). Al final de la primera temporada de crecimiento después de la primera quema en 2013 , el peso seco presentó el mayor incremento en los mesocosmos sometidos a tratamiento por fuego $(1.48 \pm 2.03 \mathrm{~g})$, seguido de los mesocosmos control ( 0.537 $\pm 0.11 \mathrm{~g}$ ). Al final de la temporada de crecimiento, después del segundo año de quema, el promedio de la producción de biomasa para todos los tratamientos fue superior a la obtenida en la temporada de crecimiento anterior $(1.79 \pm .85 \mathrm{~g})$; sin embargo, no se presentaron diferencias significativas entre los tratamientos de quema por una ocasión, dos ocasiones y el control $\sin$ quema $(Z=1.52 ; p=0.128 ; n=13)$.

\section{DISCUSIÓN}

Phragmites australis es considerada como una especie con gran capacidad de invasión de hábitat, pues responde positivamente a incrementos en las concentraciones de nutrientes y de salinidad. Por lo anterior, Price et al. (2014) indican que el monitoreo de esta especie es fundamental para el buen manejo de humedales, ya que su capacidad de invasión depende sobre todo de la dispersión de fragmentos (Kettenring et al., 2016). A pesar de lo citado, los resultados de esta investigación indican que incluso después de incendios que eliminan el dosel de especies nativas, la supervivencia y crecimiento de los fragmentos de $P$. australis fue muy baja. Resultados similares fueron reportados por Montejo-Mayo et al. (2015).

Cabe mencionar que la mayor parte del crecimiento de $S$. americanus se presentó de mayo a julio, pues en este último mes alcanzó la máxima densidad de toda la temporada al cerrar el dosel y cubrir a $P$. australis. Este proceso de crecimiento de $S$. americanus fue aún más rápido durante el segundo año en que se aplicó el tratamiento de fuego a algunas de las unidades experimentales, lo cual ocasionó que los individuos de $P$. australis presentaran alturas menores que durante el primer año. Respecto a la biomasa, ésta fue similar para los individuos de Phragmites australis independientemente de si crecieron en mesocosmos quemados o no quemados, lo que sugiere que las diferencias de altura responden a que las plantas en los mesocosmos control se encontraban etioladas como respuesta al ambiente lumínico (Monsi \& 

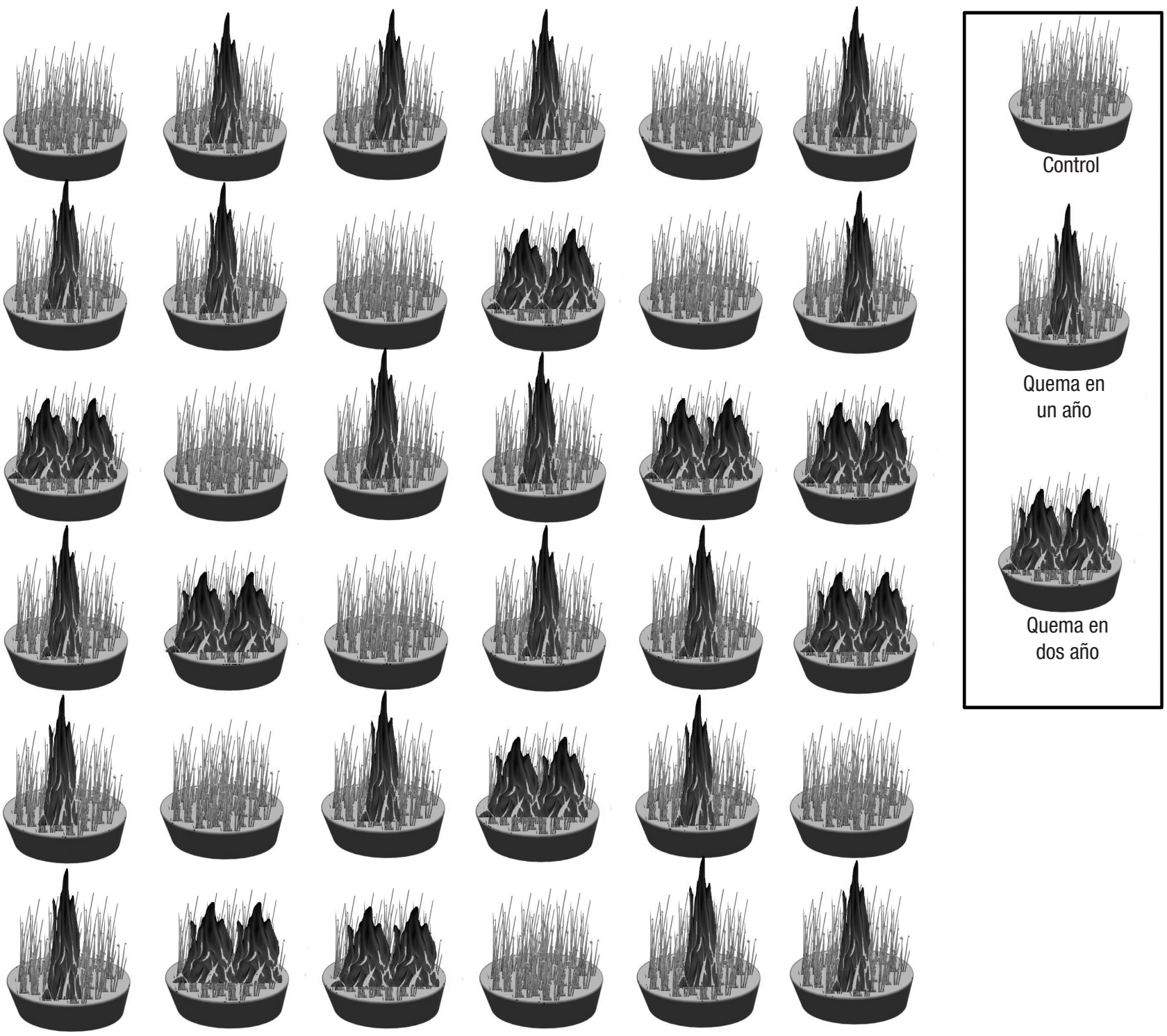

Figura 3. Esquema del experimento completo y la asignación de los mesocosmos a los diferentes tratamientos de fuego en el dosel de Schoenoplectus americanus (Pers.) Volkart ex Schinz \& R. Keller. Después del segundo año de quema, 18 mesocosmos fueron quemados una sola vez, 9 fueron quemados dos veces y 9 no fueron quemados.

Saeki, 2005). En este experimento, las condiciones más estresantes para la especie invasora, $P$. australis, se dieron en los mesocosmos control, dado que nunca perdieron el dosel. En cambio, en los mesocosmos sometidos a fuego se generó un periodo en el que el dosel estuvo ausente, lo que permitió que al menos unos pocos individuos de Phragmites australis se establecieran y lograran desarrollar un tamaño considerable para crecer en mejores condiciones, antes de que se volviera a cerrar el dosel de $S$. americanus.

El presente estudio sugiere que se necesita de más de un evento de disturbio por fuego o una acción combinada con otros tipos de disturbios para alterar la dinámica entre Schoenoplectus americanus y Phragmites australis y permitir que la segunda se establezca y al- cance un tamaño que le permita convertirse en dominante. Al parecer, las altas tasas de crecimiento de $S$. americanus y el dosel denso que desarrolla limitan la disponibilidad de luz para el establecimiento de fragmentos vegetativos de $P$. australis. Reducir la disponibilidad de luz mediada por las características del dosel de las especies nativas también reduce el establecimiento de otra especie invasora de humedales como Phalaris arundinacea L. (Lindig-Cisneros \& Zedler, 2002a; LindigCisneros \& Zedler, 2002b).

Cuando se transporta suelo de humedales en donde crece $P$. australis, se puede favorecer la invasión, debido a que los fragmentos se establecen exitosamente en ausencia de competencia con otras especies (Juneau \& Tarasoff, 2013). De hecho, aunque en algunos lugares 

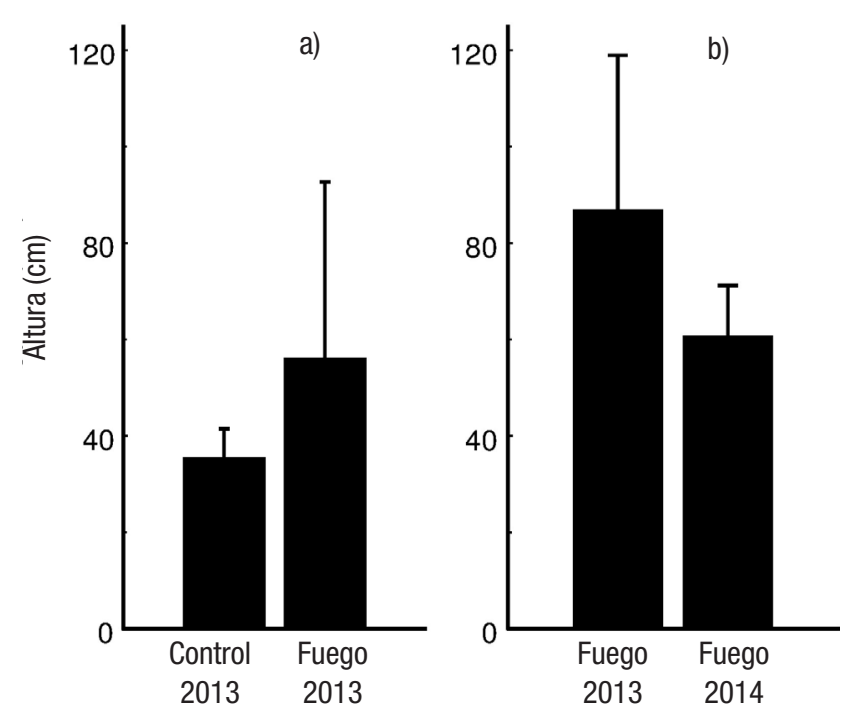

Figura 4a-b. Longitud máxima de la ramificación de Phragmites australis (Cav.) Trin ex Stuedel en un experimento de mesocosmos realizado en las instalaciones del Instituto de Investigaciones en Ecosistemas y Sustentabilidad (Morelia, Michoacán, México) en el que los tratamientos fueron: quema por un año en 2013 (a), o por dos años en 2013 y 2014 (b) durante dos estaciones de crecimiento. Las barras representan la desviación estándar.

las semillas producen un gran número de plántulas, su supervivencia es muy baja y la mayoría de las plantas que sobreviven después de dos años se generan a través de fragmentos (Albert et al., 2015), lo que indica la importancia de controlar su dispersión por este medio.

Phragmites australis, conocida como carrizo común, se considera una especie invasora traslocada y de extensa distribución en el país (Bonilla-Barbosa \& Santa María, 2014). Lowe et al. (2004) la catalogan entre las 100 especies invasoras más dañinas del mundo. Es una fuerte competidora que elimina a otras especies del humedal, provee poco alimento o refugio para especies silvestres y es capaz de eliminar diversos hábitats en canales y charcas relevantes para invertebrados, peces y aves silvestres. Al descomponerse puede elevar rápidamente el nivel del suelo y reducir la inundación y los nutrientes disponibles, lo cual le da aun mayor superioridad competitiva (U.S. Fish \& Wildlife Service, 2016). Por lo anterior, es de gran importancia para la conservación de los humedales del Altiplano mexicano conocer su capacidad de invasión, a partir de fragmentos, y su interacción con las especies nativas hidrófitas.

En términos de manejo, en humedales en donde Phragmites australis ya está presente pero que cuentan con una cobertura dominada por especies nativas, la cosecha de esta especie invasora es una estrategia efectiva (Escutia-Lara et al., 2012). De acuerdo con lo anterior y con los resultados obtenidos en este estudio, la cosecha de $P$. australis en sitios que no han sufrido efectos del fuego puede generar fragmentos que prácticamente no tienen posibilidades de establecerse, incluso si la cosecha se lleva a cabo después de eventos aislados o poco recurrentes de fuego, la posibilidad es muy baja.

\section{AGRADECIMIENTOS}

Deseamos agradecer el financiamiento otorgado por la DGAPA-UNAM a través del proyecto PAPIIT IN203316. Ma. E. Páramo-Pérez desea agradecer al Conacyt por la beca otorgada para los estudios de doctorado y al Posgrado en Ciencias Biológicas de la Universidad Nacional Autónoma de México. Este estudio es parte de los requisitos de titulación del posgrado en Ciencias Biológicas de la Universidad Nacional Autónoma de México.

\section{REFERENCIAS}

Albert, A., J. Brisson, F. Belzlle, J. Turgeon \& C. Lavole. 2015. Strategies for a succesful plant invasion: the reproduction of Phragmites australis in north-eastern North America. Journal of Ecology 103: 1529-1537.

BART, D. \& J. M. HaRTAM. 2003. The role of large rhizome dispersal and low salinity windows in the establishment of common reed, Phragmites australis, in salt marshes: New links to human activities. Estuaries 26: 436-443. D0I:10.1007/BF02823720

Bisson, P. A., B. E. Rieman, C. Luce, P. F. Hessburg, D. C. Lee, J. L. Kershner, G. H. Reeves \& R. E. Gresswell. 2003. Fire and aquatic ecosystems of the western USA: current knowledge and key questions. Forest Ecology and Management 178: 213-229. D0I:10.1016/S03781127(03)00063-X.

Bixby, R., S. D. Cooper, R. E. Gresswell, L. E. Brown, C. N. Dahm \& K. A. Dwire. 2015. Fire effects on aquatic ecosystems: an assessment of the current state of the science. Freshwater Science 34: 1340-1350.

Bonilla-Barbosa J. R. \& B. Santamaría Araúz. 2014. Plantas acuáticas exóticas y traslocadas invasoras. In: Mendoza, R. y P. Koleff (Coords.). Especies acuáticas invasoras en México. Comisión Nacional para el Conocimiento y Uso de la Biodiversidad (Conabio), México. pp. 223-248

Constanza, R., R. D’Arge., R. de Groot, S. Farber, M. Grasso, B. Hannon, K. Limburg, S. Naeem, R. V. O'Neill, J. Paruelo, R. G. Raskin, P. Sutton \& M. van der Belt. 1997. The value of the world ecosystem services and natural capital. Ecological Economics 1: 335-361.

Crawley, M. 2007. The R Book. John Wiley \& Sons Ltd, England. 949 p.

DAVID, P. 1996. Changes in plant communities relative to hydrologic conditions in the Florida Everglades. Wetlands 16: 15-23. D0l:10.1007/ BF03160642

Del Val, E., A. L. Pacheco \& R. Lindig-Cisneros. 2012. Mecanismos de persistencia de Phragmites australis en el manantial de La Minzita. Biológicas 14: 18-22.

Escutia-Lara, Y., S. Lara-Cabrera \& R. Lindig-Cisneros. 2009. Efecto del fuego y dinámica de las hidrófitas emergentes en el humedal de la Mintzita, Michoacán, México. Revista Mexicana de Biodiversidad 80: 771-778.

Escutia-Lara, Y., S. Lara-Cabrera, M. Gómez-Romero \& R. Lindig-Cisneros. 2012. Common reed (Phragmites australis Cav. Trin ex. Steud.) harvest as a control method in a Neotropical wetland in Western México. Hidrobiológica 22: 125-131 
Escutia-Lara, Y., S. Lara-Cabrera, M. Gómez-Romero \& R. Lindig-Cisneros. 2012. Dinámica de Phragmites australis y Schoenoplectus americanus en respuesta a la adición de fósforo y nitrógeno en humedales experimentales. Botanical Sciences 90: 459-467.

Hall, S., R. Lindig-Cisneros \& J. ZedLer. 2008. Does harvesting sustain plant diversity in central Mexican wetlands? Wetlands 28: 776-792. DOI:10.1672/07-231.1

JuneaU, K. J. \& C. S. Tarasoff. 2013. The seasonality of survival and subsequent growth of common reed (Phragmites australis) rhizome fragments. Invasive Plant Science and Management 6: 79-86.

Korsgaard, L. \& J. S. Schou. 2010. Economic valuation of aquatic ecosystem services in developing countries. Water Policy 12: 20-31. D0l:10.2166/wp.2009.124

Kost, M. A. \& D. SteVEn. 2001. Plant community responses to prescribed burning in Wisconsin sedge meadows. Natural Areas Journal 687: 36-45.

Landgrave, R. \& P. Moreno-Casasola. 2012. Evaluación cuantitativa de la pérdida de humedales en México. Investigación Ambiental 4 (1): 35-51.

López-Rosas, H. \& P. Moreno-Casasola. 2012. Invader versus natives: Effects of hydroperiod on competition between hydrophytes in a tropical freshwater marsh. Basic and Applied Ecology 13: 40-49.

Lindig-Cisneros, R. \& J. B. Zedler. 2002a. Phalaris arundinacea seedling establishment: effects of canopy complexity in fen, mesocosm, and restoration experiments. Canadian Journal of Botany 80: 617-624.

Lindig-Cisneros, R. \& J. B. ZedLer. 2002b. Relationships between canopy complexity and germination microsites for Phalaris arundinacea $\mathrm{L}$. Oecologia 133: 159-167.

Lowe, S., M. Browne, S. Boudjelas \& M. De Poorter. 2004. 100 de las especies exóticas invasoras más dañinas del mundo. Una selección del Global Invasive Species Database. Grupo Especialista de Especies Invasoras (GEEI), Comisión de Supervivencia de Especies (CSE), Unión Mundial para la Naturaleza (UICN): 1-12.

meA (Millennium Ecosystem Assessment). 2005. Ecosystems and Human Well-Being. Synthesis Island Press: 1-36.

Miller, S. J., K. J. Ponzio, M. A. Lee, L. W. Keenan \& S. R. Mleer. 1998. The use of fire in wetland preservation and restoration: are there risks? In: Editors: Pruden, T. L. \& L. A. Brennan (Eds.). Fire in ecosystem management: shifting the paradigm from suppression to prescription. Edition: No.20, Tall Timbers Research Station, Tallahassee, Florida, pp. 127-139.

Miтsch, W. J. \& J. G. GosseLink. 2000. Wetlands. John Wiley and Sons Inc. Nueva York. 920 p.

MonsI, M \& T. SAEKI. 2005. On the Factor Light in Plant Communities and its Importance for Matter Production. Annals of Botany 95: 549-56.

Montejo-Mayo, W., E. del Val, M. Gomez-Romero, E. de la Barrera \& R. LindigCISNEROS. 2015. Interactions between dominant hydrophytic species of the wetlands of western Mexico mediated by fire and nitrate concentration. Polibotánica 40: 153-161.

Moreno-Casasola, P., E. Cejudo-Espinosa, A. Capistrán-Barradas, D. InfanteMata, H. López-Rosas, G. Castillo-Campos, J. Pale-Pale \& A. CamposCascaredo. 2010. Composición florística, diversidad y ecología de humedales herbáceos emergentes en la planicie costera central de Veracruz, México. Boletín Sociedad Botánica de México 87: 29-50.

Newman, S., J. B. Grace \& J.W. Koebel. 1996. Effects of nutrients and Hydroperiod on Thypa, Cladium, and Eleocharis: Implications for Everglades Restoration. Ecological Applications 6: 774-783.

Norton, D. A. \& P. J. De Lange. 2002. Fire and vegetation in a temperate peat bog: Implications for the management of threatened species. Conservation Biology 17: 138-148. D0l:10.1046/j.15231739.2003.01131.x

Pettit, N. E. \& R. J. Naiman. 2007. Fire in the riparian zone: characteristics and ecological consequences. Ecosystems 10: 673-687. DOI:10.1007/s10021-007-9048-5.

Price, A.L., J.B. Fant \& D. J. Larkin. 2014. Ecology of Native vs. Introduced Phragmites australis in Chicago-area Wetlands. Wetlands 34: 369-377.

R Core Team. 2017. R: A language and environment for statistical computing. R Foundation for Statistical Computing, Vienna, Austria. URL https://www.R-project.org/.

Rice, D., J. Rooth \& J. Court Stevenson. 2000. Colonization and invasion of Phragmites australis in upper Cheasepeake bay tidal marshes. Wetlands 2: 280-299. D0I:10.1672/0277-5212(2000)020[0280:CA EOPA]2.0.CO;2

Swearinger, J. \& K. Saltonstall. 2010. Phragmites field guide: distinguishing native and exotic forms of common reed (Phragmites australis) in the United States. Weeds Gone Wild, Plant Conservation Alliance. Disponible en línea en: http://www.nps.gov/plants/alien/ fact/pdf/phau1-powerpoint.pdf (consultado el 12 de noviembre de 2016).

U.S. Fish and Wildiffe Service. 2016. Phragmites australis fact sheet. Disponible en línea en: http://www.fws.gov/gomcp/pdfs/phragmitesqa_factsheet.pdf (consultado el 15 de noviembre del 2016).

ZedLeR, J. B. \& S. KerCher. 2004. Causes and consequences of invasive plants in wetlands: opportunities, opportunists, and outcomes. Critical Reviews in Plant Science 23: 431-452.

Zaman, B. \& M. McKee. 2016. Life on the edge: reproductive mode and rate of invasive Phragmites australis patch expansion. Biological Invasions 18: 2475-2495.

ZedLer, J. B. \& S. KeRCHER. 2005. Wetland resources: status, trends, ecosystem services, and restorability. Annual Review of Environment and Resources 30: 39-74. 